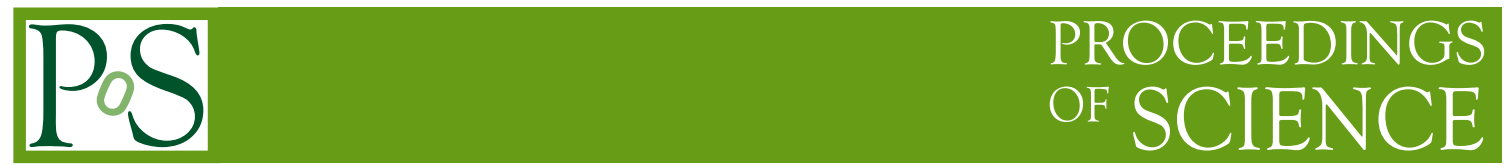

\title{
Meson Resonances on the Lattice
}

\section{Robert G. Edwards*}

Thomas Jefferson National Accelerator Facility

E-mail: edwards@jlab.org

There has been recent, significant, advances in the determination of the meson spectrum of QCD. Current efforts have focused on the development and application of finite-volume formalisms that allow for the determination of scattering amplitudes as well as resonance behavior in coupled channel systems. I will review some of these recent developments, and demonstrate the viability of the method in meson systems.

The 8th International Workshop on Chiral Dynamics, CD2015 ***

29 June 2015 - 03 July 2015

Pisa,Italy

${ }^{*}$ Speaker. 


\section{Introduction}

Spectroscopy has played, and continues to play, a pivotal role in the development of our understanding of the strong interaction. For many years, the simplicity of the quark model, in which every meson is made out of a quark and an antiquark, and every baryon out of three quarks, with quarks in a few different flavors, encapsulated our knowledge of the spin, parity and flavor properties of all hadrons. Indeed, this simple picture was a key driver in the proposal that QCD (Quantum Chromo-Dynamics) is the theory underlying the strong interactions. Nevertheless, there was always the suspicion that other quark and gluon configurations with a wider set of spin, parity and flavor quantum numbers might well be possible. Without a detailed understanding of the mechanism of color confinement, QCD led us to expect a variety of color singlet hadrons, not just $\bar{q} q$ and $q q q$ would exist. Lattice QCD calculations have reinforced such expectations with predictions of glueballs, and hybrid mesons and baryons in which the minimal quark content is supplemented with an excitation of the gluonic field. While hints of glueballs and hybrids have been found experimentally, none has been overwhelmingly convincing. The discovery of states of hidden heavy flavor, first charmonium and then bottomonium, for long confirmed that a simple non-relativistic modeling of the interquark potential described these states remarkably well as nothing more than $q \bar{q}$ with $q=c$, or $b$. It is in this seemingly understood sector of the hadron spectrum that surprising discoveries have recently been made. The discovery of not just one, but very many new states that do not fit the simple $q \bar{q}$ pattern, has caused a storm of interest in this novel spectroscopy. At the same time new experiments, such as the GlueX experiment in Jefferson Lab's new Hall D, will search for exotic states in the light quark sector. There is intense theoretical activity to explain these new experimental observations, as well as making predictions for future observations.

\section{Lattice QCD and hadron scattering}

Lattice QCD offers the possibility of computing hadron properties directly from QCD, through numerical computation of the QCD path integral under the controlled approximation of a finite, discretized, space-time grid. While a program of computations of ever increasing precision of simple properties of stable hadrons, such as masses and decay constants, has attracted the attention of a large community of researchers for many years, the challenging problem of determining the $e x$ cited resonance spectrum of QCD has only recently been seriously addressed. In the past few years significant progress has been made determining hadron scattering amplitudes directly from QCD using lattice methods. As well as being the basic physical observables extracted from accelerator experiments, it is from the energy dependence of hadron scattering amplitudes that we may determine the resonant content of the theory. If we are to deduce the nature of resonances within QCD it must be via scattering amplitudes.

Scattering amplitudes cannot be directly extracted from the Euclidean correlation functions computed in lattice QCD, but rather they can be determined by considering the discrete spectrum of eigenstates in the finite volume defined by the boundary of the lattice. The relationship between this spectrum and the scattering amplitudes of the theory in infinite volume was first laid out by Lüscher in 1990 [1], with elaborations of the formalism dealing with frames moving with respect to the lattice following later $[2,3]$. The formalism, in the simplest case of elastic scattering in 
a single partial-wave, $\ell$, produces the result $\cot \delta_{\ell}(E)=F(E, L)$, which indicates that the elastic scattering phase-shift at energy $E, \delta_{\ell}(E)$, is related to a known kinematical function of energy and volume, $F(E, L)$. In practice, if we determine the discrete spectrum of states in a lattice QCD calculation in volume $L \times L \times L$, then for each energy level, $E_{n}(L)$, we deduce the phase-shift at that energy, $\delta_{\ell}\left(E_{n}\right)$. By extracting many excited energy levels, computing in different volumes and/or determining the spectrum in moving frames, we should be able to map out the energy-dependence of the elastic scattering phase-shift.

While the formalism has been known for more than two decades, it is only recently that the lattice technology has come together to make these calculations feasible in practice. The Hadron Spectrum Collaboration has been at the forefront of these developments. It is now possible to reliably extract the excited spectrum of states from two-point correlation functions of the form $C_{i j}(t)=\left\langle 0\left|\mathscr{O}_{i}(t) \mathscr{O}_{i}(0)\right| 0\right\rangle$, where $\mathscr{O}_{i}$ are composite operators with the quantum numbers of mesons, constructed from the quark and gluon fields of QCD. In particular we have developed a large basis of such operators resembling single mesons for a wide range of possible $J^{P C}[4,5,6]$, as well as those resembling multi-meson constructions like $\pi \pi$ [7]. By evaluating correlation functions featuring many such operators, we are able to form them into a matrix, whose entries are $C_{i j}(t)$, which can be diagonalized to determine the best estimate, in a variational sense, for the spectrum. Evaluating the large number of correlation functions in a computationally efficient manner is made possible by a technique known as distillation [8], in which the construction of operators factorizes from the propagation of quarks, combined with our pioneering applications of Graphics Processing Units to the computations of the quark propagation. This combination of novel approaches also made practical the evaluation of diagrams featuring quark annihilation, required in calculations featuring isoscalar states and in most scattering calculations. Taken together this progress has led us to a current situation where spectrum extractions of the type illustrated in Figure 1 are routine - the discrete spectrum, in units of the lattice spacing, determined on three lattice volumes $\left(16^{3}, 20^{3}, 24^{3}\right)$ is shown, following a diagonalization of a correlation matrix, which on the largest volume has dimension 27 including a number of meson-meson-like operators resembling $\pi K$ and $\eta K$ constructions.

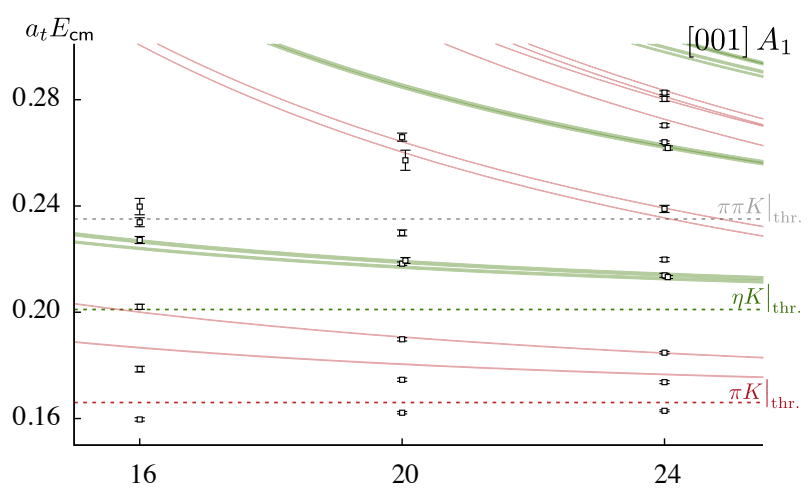

These technical advances have not only been applied to problems of scattering of hadrons, but
Figure 1: Example of an excited state spectrum extracted from a contemporary lattice QCD calculation $[9,10]$. The energy in lattice units computed on three lattice volumes is shown. In the $24^{3}$ case, 12 states with statistical precision under $1 \%$ are presented, extracted from the diagonalisation of a matrix of correlation functions built using 27 operators. The colored lines show the distribution of $\pi K, \eta K$ states expected in a theory of non-interacting hadrons - deviations from these lines indicate the effect of interactions. Calculation performed on lattices where $m_{\pi}=$ $391 \mathrm{MeV}$ 
also in a number of other calculations performed by the Hadron Spectrum Collaboration, including the determination of the gross features of excited light meson spectrum [4, 5], the flavor structure of isoscalar mesons [11, 12], the excited spectrum of charmonium and charmed mesons [13, 14], decay constants of excited pions [15], and the spectrum of baryons [16, 17, 18, 19, 20].

An illustrative case of the computation of elastic hadron scattering amplitudes is furnished by $\pi \pi$ in isospin- $1 P$-wave, in which we expect there to be a low-lying $\rho$ resonance. This channel has been considered in several recent lattice calculations [21, 22, 23, 24, 25, 26]. The Hadron Spectrum Collaboration calculation [21, 27] considered several moving frames and lattice volumes with quark masses such that the pion has a mass of $391 \mathrm{MeV}$ down to $236 \mathrm{MeV}$. It was found that the resulting discrete spectrum led to the elastic phase-shift shown in Figure 2. A clear mappingout of the resonant lineshape is observed, leading to a determination of the mass and width of the $\rho$ in line with expectations at these artificially heavy quark masses.

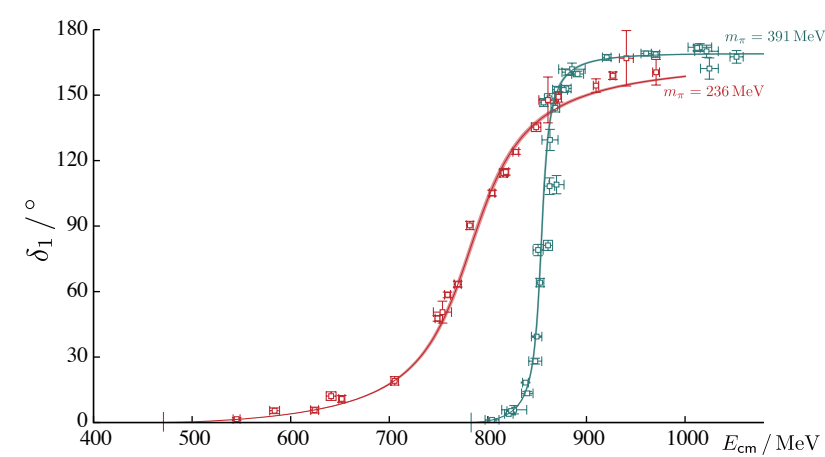

Figure 2: Isospin-1, $P$-wave phase-shift in the $\pi \pi$ elastic scattering region (below $K \bar{K}$ threshold). Phase-shift points mapped from finite-volume energies in several volumes and moving frames using the Lüscher formalism. Curve shows a Breit-Wigner fit to the energy dependence which shows a clear resonant lineshape. The mass and width of the resonance are in line with expectations for a calculation with $m_{\pi}=391 \mathrm{MeV}$ and $m_{\pi}=236 \mathrm{MeV}$. Figure taken from Ref. [27].

Since most hadron resonances decay into more than one final state, we must consider situations beyond elastic scattering and allow for coupled-channel amplitudes. The relationship between the discrete spectrum in a finite-volume and coupled-channel amplitudes has been presented recently $[28,29,30,31,32,33,34]$, the same result being derived within a number of formal approaches to quantum field theory. The finite-volume spectrum is given by those energies satisfying the equation, $0=\operatorname{det}\left[t^{-1}(E)+i \rho(E)-\rho(E) F(E, L)\right]$, where the scattering matrix has elements $t_{i j}(E)$ indexed according to the kinematically open scattering channels, $i=1 \ldots N$. $\rho_{i}(E)=2 k_{i}(E) / E$ is the phase-space for channel $i$, and $F_{i j}(E, L)$ is a known kinematic function, diagonal in the channel space. Unitarity and time-reversal invariance constrain the $t$-matrix such that $\frac{1}{2} N(N+1)$ real numbers are needed to completely describe it at any given energy, which presents a problem of application for this formula: for each energy level, $E_{n}(L)$, determined in a lattice QCD calculation, we have one equation in $\frac{1}{2} N(N+1)$ unknowns, which clearly cannot be solved.

A successful approach to this problem is to parameterize the energy dependence of the scattering $t$-matrix and to describe the entire finite-volume spectrum, over a range of energies, in one go, by varying adjustable parameters. In fact, the cubic symmetry of the boundary, and the even further reduced symmetry in a moving frame, means that the partial-wave basis is not diagonal, and the determinant in the above expression is also evaluated over multiple partial waves. This provides an additional technical complication, which can be addressed by parameterizing each partial wave separately. 
The first application of this coupled-channel formalism to an explicit lattice QCD computation was recently carried out by the Hadron Spectrum Collaboration $[9,10]$. This study considered the isospin-1/2 coupled $\pi K, \eta K$ sector in partial waves $J^{P}=0^{+}, 1^{-}, 2^{+}$in which kaon resonances are expected to feature. The calculation was performed in three volumes, considering multiple moving frames, with spectra like those presented in Figure 1 being determined. Using relatively simple $K$-matrix parameterizations to describe the two-channel scattering system and taking advantage of over one hundred energy levels to constrain the parameterizations, it was possible to extract the amplitudes shown in Figure 3.
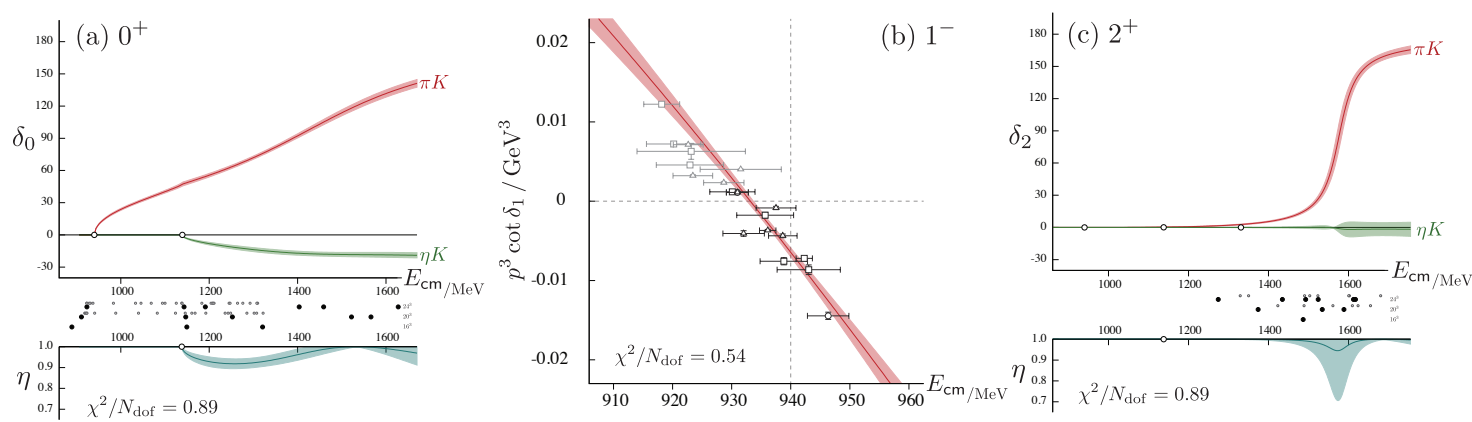

Figure 3: Scattering amplitudes for the strangeness-1, isospin-1/2 channel in three partial waves. (a) The coupled $\pi K, \eta K S$-wave presented via two phase-shifts (upper panel) and an inelasticity (lower panel). (b) $\pi K$ scattering near threshold in the $P$-wave - zero crossing of $p^{3} \cot \delta$ indicates the position of a $K^{\star}$ boundstate. (c) The $D$-wave scattering amplitude, showing a clear narrow resonance which we find is coupled strongly to only $\pi K$. Small $\chi^{2}$ values indicate a good description of the finite-volume spectra (shown by the dots in the central panels) using simple $K$-matrix parameterizations. Taken from Ref. [9, 10]

The parameterizations of the scattering $t$-matrix may be analytically continued to complex values of the energy, and their singularity structure explored. As well as the branch-cut singularities required by unitarity, we may search for pole singularities which correspond to bound-states and resonances. In the calculation presented in Refs. [9, 10], which used a pion mass of $391 \mathrm{MeV}$, the $1^{-}$partial wave was found to contain a pole on the real energy axis, on the physical sheet, just below threshold, corresponding to a $K^{\star}$ manifested as a bound-state - as the pion mass is decreased, this would be expected to become a resonance. The $2^{+}$partial wave amplitude contained a pole lying slightly away from the real energy axis on unphysical sheets, corresponding to a narrow tensor resonance, $K_{2}^{\star}$. Examining the residues of the $t$-matrix at the pole position allowed the extraction of the couplings of this resonance to the $\pi K$ and $\eta K$ final states. The $0^{+}$partial wave showed a richer singularity structure, with a pole above $\pi K$ threshold, lying far from the real energy axis, likely corresponding to a broad scalar resonance, $K_{0}^{\star}$, and in addition, a pole on the real axis below $\pi K$ threshold, lying on unphysical sheets, which could be interpreted as a "virtual bound state". A possible identification of this state with the expected behavior of the $\kappa$ resonance as the pion mass is increased was made.

The first extraction of coupled-channel scattering amplitudes reported in $[9,10]$ was mostly limited to the energy region below the opening of the $\pi \pi K$ threshold. We chose not to include operators resembling three-meson constructions in our correlator matrix, so the spectrum above the threshold is unlikely to be complete. The lattice technology to evaluate correlation functions 
using three-meson and higher operators does exist, and extraction of the full spectrum is quite feasible, but what is lacking currently is the formalism relating the finite-volume spectrum to the three-meson scattering amplitudes. Having such a formalism increasingly becomes a necessity as the pion mass is reduced toward its physical value.

The relativistic three-body scattering problem is challenging even in infinite volume. Nevertheless, there has been recent significant progress in the development of this formalism in a finite volume in a relativistic quantum field theory [35], following earlier work done in a NREFT framework [36]. A generalization of Lüscher's two-particle quantization condition [1] has been derived for a massive scalar field restricted by a $Z_{2}$ (G-parity-like) symmetry such that vertices involving even numbers of particles are present [35]. The formalism, at present, does not allow for a subtwo-body resonance, but it is believed this issue can be resolved. What is clear is that techniques developed in the context of analysis of experimental three-meson final states, for example by the Joint Physics Analysis Center (JPAC) and others, can prove helpful for the lattice investigations. Since ultimately we should be using the same scattering amplitudes to describe the experimental data (in infinite volume) and the QCD spectra (in finite volume), there is an increased need for the involvement in lattice QCD projects of scientists with expertise in $S$-matrix theory.

Other techniques are at our disposal. Computing the scattering amplitudes as a function of pion mass permits a qualitative description of the relevance of the various open channels as the light quark mass varies. The amplitudes can also be used to constrain phenomenological effective field theory models. For example, non-perturbative matrix elements that appear in effective field theories for the $X$ (3872) meson [37] can be extracted from the amplitudes at unphysical pion masses and used in EFT calculations with the physical pion mass. These matrix elements can be compared to those computed from lattice calculations [38, 39, 40, 41, 42, 43].

\section{Future Directions}

The big picture from the initial lattice calculations with only single particle constructions suggests that there is a rich spectrum of hadron states, consistent in pattern with the non-relativistic quark model, but with important additions. Namely, there is a prediction of a set of exotic mesons and also non-exotic hybrid mesons and baryons that could be observed in new experiments. These observation carry over to the light and strange quark sector, as well as in the charm quark meson spectrum. There appears to be an energy scale of about $1.3 \mathrm{GeV}$ associated with $8_{F}$ color-octet configurations of gluon with $1^{+-}$quantum numbers. It should be emphasized, though, that this is a picture. While the results have been determined non-perturbatively from $\mathrm{QCD}$, they are still incomplete. What is missing are the predictions for the branching fractions of these states determined via the scattering amplitudes of the decay products.

The determination of decay information is a main direction for future lattice calculations of the spectrum. At higher energies, many-body decay modes must be disentangled, which of course, is quite a complicated undertaking. The obvious first place for investigations is the low-energy sector of QCD. This sector is interesting in its own right. The nature of two pion scattering and the production of a $\sigma$ resonance has long been controversial. In fact, knowledge of this sector is important for higher energies. The $\sigma$ resonance should occur in $S$-wave scattering of $\pi \pi$. It is not definitively known how resonances should develop as the quark mass is decreased near an $S$-wave 
threshold, although there are suggestions $[44,45]$. As higher energy resonances can have $S$-wave as well as higher wave contributions, understanding the partial wave decomposition of resonances is crucial. Thus, understanding a "simpler" sector is vital.

Of course, we desire these lattice calculations in the physical limit of QCD. Part of the answer why this is not today is cost, but that is not the main reason. In the physical limit of QCD, the simplest $\rho$ resonance is well above $4 \pi$ threshold, and a completely rigorous treatment of the decay of this state should involve a four-body formalism. This is a tall-order. Thus, the near term direction for the field is to build a first-principles understanding of the interactions of QCD in the light, strange and charm sector of QCD, and explore the resonant content as the light quark mass is varied.

\section{References}

[1] Martin Lüscher. Two particle states on a torus and their relation to the scattering matrix. Nucl.Phys., B354:531-578, 1991.

[2] K. Rummukainen and Steven A. Gottlieb. Resonance scattering phase shifts on a nonrest frame lattice. Nucl.Phys., B450:397-436, 1995.

[3] C.h. Kim, C.T. Sachrajda, and Stephen R. Sharpe. Finite-volume effects for two-hadron states in moving frames. Nucl.Phys., B727:218-243, 2005.

[4] Jozef J. Dudek, Robert G. Edwards, Michael J. Peardon, David G. Richards, and Christopher E. Thomas. Highly excited and exotic meson spectrum from dynamical lattice QCD. Phys.Rev.Lett., 103:262001, 2009.

[5] Jozef J. Dudek, Robert G. Edwards, Michael J. Peardon, David G. Richards, and Christopher E. Thomas. Toward the excited meson spectrum of dynamical QCD. Phys.Rev., D82:034508, 2010.

[6] Christopher E. Thomas, Robert G. Edwards, and Jozef J. Dudek. Helicity operators for mesons in flight on the lattice. Phys.Rev., D85:014507, 2012.

[7] Jozef J. Dudek, Robert G. Edwards, and Christopher E. Thomas. S and D-wave phase shifts in isospin-2 pi pi scattering from lattice QCD. Phys.Rev., D86:034031, 2012.

[8] Michael Peardon, John Bulava, Justin Foley, Colin Morningstar, Jozef Dudek, Robert G. Edwards, Bálint Joó, Huey-Wen Lin, David G. Richards, and Keisuke Jimmy Juge. A Novel quark-field creation operator construction for hadronic physics in lattice QCD. Phys.Rev., D80:054506, 2009.

[9] Jozef J. Dudek, Robert G. Edwards, Christopher E. Thomas, and David J. Wilson. Resonances in coupled $\pi K-\eta K$ scattering from quantum chromodynamics. Phys.Rev.Lett., 113(18):182001, 2014.

[10] David J. Wilson, Jozef J. Dudek, Robert G. Edwards, and Christopher E. Thomas. Resonances in coupled $\pi K, \eta K$ scattering from lattice QCD. Phys. Rev., D91(5):054008, 2015.

[11] Jozef J. Dudek, Robert G. Edwards, Bálint Joó, Michael J. Peardon, David G. Richards, and Christopher E. Thomas. Isoscalar meson spectroscopy from lattice QCD. Phys.Rev., D83:111502, 2011.

[12] Jozef J. Dudek, Robert G. Edwards, Peng Guo, and Christopher E. Thomas. Toward the excited isoscalar meson spectrum from lattice QCD. Phys.Rev., D88(9):094505, 2013.

[13] Liuming Liu, Graham Moir, Michael Peardon, Sinéad M. Ryan, ChristopherE. Thomas, Pol Vilaseca, Jozef J. Dudek, RobertG. Edwards, Bálint Joó, and DavidG. Richards. Excited and exotic charmonium spectroscopy from lattice QCD. JHEP, 1207:126, 2012. 
[14] Graham Moir, Michael Peardon, Sinead M. Ryan, Christopher E. Thomas, and Liuming Liu. Excited spectroscopy of charmed mesons from lattice QCD. JHEP, 1305:021, 2013.

[15] Ekaterina V. Mastropas and David G. Richards. Decay constants of the pion and its excitations on the lattice. Phys.Rev., D90(1):014511, 2014.

[16] Robert G. Edwards, Jozef J. Dudek, David G. Richards, and Stephen J. Wallace. Excited state baryon spectroscopy from lattice QCD. Phys.Rev., D84:074508, 2011.

[17] Jozef J. Dudek and Robert G. Edwards. Hybrid Baryons in QCD. Phys.Rev., D85:054016, 2012.

[18] Robert G. Edwards, Nilmani Mathur, David G. Richards, and Stephen J. Wallace. Flavor structure of the excited baryon spectra from lattice QCD. Phys.Rev., D87(5):054506, 2013.

[19] M. Padmanath, Robert G. Edwards, Nilmani Mathur, and Michael Peardon. Spectroscopy of triply-charmed baryons from lattice QCD. Phys.Rev., D90(7):074504, 2014.

[20] M. Padmanath, Robert G. Edwards, Nilmani Mathur, and Michael Peardon. Spectroscopy of doubly-charmed baryons from lattice QCD. Phys. Rev., D91(9):094502, 2015.

[21] Jozef J. Dudek, Robert G. Edwards, and Christopher E. Thomas. Energy dependence of the $\rho$ resonance in $\pi \pi$ elastic scattering from lattice QCD. Phys.Rev., D87(3):034505, 2013.

[22] S. Aoki, M. Fukugita, K-I. Ishikawa, N. Ishizuka, K. Kanaya, Y. Kuramashi, Y. Namekawa, M. Okawa, K. Sasaki, A. Ukawa, and T. Yoshié. Lattice QCD Calculation of the rho Meson Decay Width. Phys.Rev., D76:094506, 2007.

[23] Xu Feng, Karl Jansen, and Dru B. Renner. Resonance Parameters of the rho-Meson from Lattice QCD. Phys.Rev., D83:094505, 2011.

[24] C.B. Lang, Daniel Mohler, Sasa Prelovsek, and Matija Vidmar. Coupled channel analysis of the rho meson decay in lattice QCD. Phys.Rev., D84(5):054503, 2011.

[25] S. Aoki, K-I. Ishikawa, N. Ishizuka, K. Kanaya, Y. Kuramashi, Y. Namekawa, M. Okawa, Y. Taniguchi, A. Ukawa, N. Ukita, T. Yamazaki, and T. Yoshié. $\rho$ Meson Decay in 2+1 Flavor Lattice QCD. Phys.Rev., D84:094505, 2011.

[26] Craig Pelissier and Andrei Alexandru. Resonance parameters of the rho-meson from asymmetrical lattices. Phys.Rev., D87(1):014503, 2013.

[27] David J. Wilson, Raul A. Briceno, Jozef J. Dudek, Robert G. Edwards, and Christopher E. Thomas. Coupled $\pi \pi, K \bar{K}$ scattering in $P$-wave and the $\rho$ resonance from lattice QCD. Phys. Rev., D92(9):094502, 2015.

[28] Song He, Xu Feng, and Chuan Liu. Two particle states and the S-matrix elements in multi-channel scattering. JHEP, 0507:011, 2005.

[29] M. Doring, Ulf-G. Meissner, E. Oset, and A. Rusetsky. Unitarized Chiral Perturbation Theory in a finite volume: Scalar meson sector. Eur.Phys.J., A47:139, 2011.

[30] Raul A. Briceno and Zohreh Davoudi. Moving Multi-Channel Systems in a Finite Volume. Phys. Rev., D88:094507, 2013.

[31] Maxwell T. Hansen and Stephen R. Sharpe. Multiple-channel generalization of Lellouch-Luscher formula. Phys.Rev., D86:016007, 2012.

[32] Peng Guo, Jozef Dudek, Robert Edwards, and Adam P. Szczepaniak. Coupled-channel scattering on a torus. Phys. Rev., D88(1):014501, 2013. 
[33] Jia-Jun Wu, T.-S.H. Lee, A.W. Thomas, and R.D. Young. Finite-volume Hamiltonian method for coupled-channels interactions in lattice QCD. Phys.Rev., C90(5):055206, 2014.

[34] Raul A. Briceno. Two-particle multichannel systems in a finite volume with arbitrary spin. Phys.Rev., D89:074507, 2014.

[35] Maxwell T. Hansen and Stephen R. Sharpe. Relativistic, model-independent, three-particle quantization condition. Phys.Rev., D90(11):116003, 2014.

[36] K. Polejaeva and A. Rusetsky. Three particles in a finite volume. Eur.Phys.J., A48:67, 2012.

[37] Eric Braaten. Galilean-invariant effective field theory for the X(3872). Phys. Rev., D91(11):114007, 2015.

[38] Jozef J. Dudek, Robert Edwards, and Christopher E. Thomas. Exotic and excited-state radiative transitions in charmonium from lattice QCD. Phys.Rev., D79:094504, 2009.

[39] Christian J. Shultz, Jozef J. Dudek, and Robert G. Edwards. Excited meson radiative transitions from lattice QCD using variationally optimized operators. Phys. Rev., D91(11):114501, 2015.

[40] Raúl A. Briceño, Maxwell T. Hansen, and Andre Walker-Loud. Multichannel $1 \rightarrow 2$ transition amplitudes in a finite volume. Phys.Rev., D91(3):034501, 2015.

[41] Raúl A. Briceño, Maxwell T. Hansen, and Andre Walker-Loud. Multichannel $1 \rightarrow 2$ transition amplitudes in a finite volume. PoS, LATTICE2014:095, 2015.

[42] Raúl A. Briceño and Maxwell T. Hansen. Multichannel 0-to-2 and 1-to-2 transition amplitudes for arbitrary spin particles in a finite volume. Phys. Rev., D92(7):074509, 2015.

[43] Raul A. Briceno, Jozef J. Dudek, Robert G. Edwards, Christian J. Shultz, Christopher E. Thomas, and David J. Wilson. The resonant $\pi^{+} \gamma \rightarrow \pi^{+} \pi^{0}$ amplitude from Quantum Chromodynamics. (to be published in Phys.Rev.Lett.), 2015.

[44] J. Nebreda and J. R. Pelaez. Strange and non-strange quark mass dependence of elastic light resonances from SU(3) Unitarized Chiral Perturbation Theory to one loop. Phys. Rev., D81:054035, 2010.

[45] C. Hanhart, J. R. Pelaez, and G. Rios. Remarks on pole trajectories for resonances. Phys. Lett., B739:375-382, 2014. 